- She forged ahead, discussing unconventional work that blends Western and traditional Maori research methods to an audience of experts from around the world. Her presentation was warmly received. Researchers bombarded her with questions about working with indigenous groups - which often face rates of drowning that are higher than national averages. More important to Phillips, however, were the eager questions and comments coming from the Maori community members whom she worked with: she had live-streamed her talk so that they could listen and weigh in. "That was a big moment for me," she says.

Back in Dunedin, Phillips is part of a group that supports young Maori researchers, who are under-represented in New Zealand science. Many countries are attempting to increase the diversity of their scientific workforces and to make the research they support better reflect the varied concerns of their populations. And the trend towards inclusion in science has also yielded benefits to the researchers who embrace it. A variety of studies have tracked different types of diversity - ethnic, gender, nationality and scientific discipline - and suggest that particularly diverse groups publish a higher number of papers and receive more citations per paper than average ${ }^{1,2}$. Diverse groups also seem to achieve better community participation when studying minority populations, and they often benefit from the different ideas and perspectives that the team members can bring.

Nature talked to three groups that have prioritized diversity in their ranks to ask about the benefits they have seen and the challenges and trade-offs they have to accept as part of the sometimes-difficult job of being inclusive (see page 149).

Although they differ greatly by geography and discipline, the groups share some key elements, including lab leaders who are directly engaged in the work, have high expectations and think that a diverse team produces the strongest research. These factors seem to be the secret sauce for these groups' notable cohesion and robust research output.

For Anne-Marie Jackson, one of Phillips's advisers, those benefits are secondary to the goal of creating science that will better serve the needs of indigenous communities. It's science's job to provide solutions for diverse communities, she says, so science must "reflect a diversity that comes from outside the ivory tower".

\section{A CULTURE OF RESPECT}

When Jackson, who studies health and physical education in Maori communities, joined the faculty at Otago in 2011, there was only one Maori graduate student in the programme. Jackson saw that as a serious problem. "The engine room of any research group, department or school is the graduate-student group," she says. So, in 2013, Jackson teamed up with Hauiti Hākopa, a Maori cultural scientist, to create Te Koronga, a programme designed specifically for Maori graduate students.

The programme's name is intended to reflect 'a desire for higher learning' and the group grounds students in Maori research methods alongside Western ones. Students learn lessons such as how to behave when asking questions in indigenous communities and how to interpret data from the 'oral literature' of traditional knowledge passed down in storytelling. Jackson usually has 10-15 honours, master's and doctoral students in the Te Koronga group. At regular Monday meetings, they speak the Maori language te reo Mãori, which many of the students are learning. The discussions help them to connect with traditional knowledge and legitimize both its place and theirs in academia, says Phillips.

Phillips's work, for example, aims to strengthen Maori cultural connections to seafaring ancestors who reached New Zealand's shores many generations ago. It includes reconnecting young people with traditional practices that elicit a strong sense of respect for the water. Phillips has found that that's often a more successful strategy to introducing water safety than, say, first handing them a life jacket.
The group's research centres on working with Maori communities to improve their well-being, not working 'on' them. This approach dovetails with recommendations made by Linda Tuhiwai Smith in her 1999 book, Decolonizing Methodologies. She argues that indigenous communities that have formerly been 'the researched' must now become 'the researchers' who set the agenda.

Such approaches promise to help fill a troubling gap in many areas of human research. For example, Esteban Burchard, Sam Oh at the University of California, San Francisco, and their collaborators showed in 2015 that fewer than $5 \%$ of federally funded studies on respiratory health in the United States represent people from minority groups ${ }^{3}$. "We're using these studies to inform policy and drug development," says Oh. "There's a problem when we take this average data that is disproportionately white and then apply it to other populations."

Jackson points out that there are other practical incentives for fostering cultural ties and a collaborative spirit with research community members. Research that solves real-world problems earns a boost in the New Zealand system of performance-based research funding. And success is measured in part through statements from community members about how well programmes support their goals.

Phillips found, also, that livestreaming her international talk kept her accountable to her community. She is on track to be the first Te Koronga student to graduate with a PhD, but all of those who have graduated with honours and master's degrees have gone on to work in research, for government agencies or in Maori community programmes.

Jackson and Phillips both stress that Te Koronga is not about lowering standards for indigenous students to enter academic careers - a frequent argument from critics of such programmes. On the contrary, it demands academic excellence. Students need that to get in the front door, says Jackson, "but that is what our communities deserve, too - to have the best possible people working with them".

\section{BORDERLESS RESEARCH}

Materials scientist Mukhles Sowwan says he doesn't actively try to recruit diverse, international researchers to his group - it just happens. In 2011, the Palestinian researcher moved from Al-Quds University in Jerusalem to the Okinawa Institute of Science and Technology Graduate University (OIST) in Japan, a fledgling start-up institute with a mandate to recruit across international borders.

"It's a unique experiment and I wanted to be part of that," Sowwan says, calling OIST "a perfect place to do research".

OIST sits on the tropical island of Okinawa, which helps it to attract scientists from around the world. Its founding board, which included heavy-hitter Nobel laureates Sydney Brenner, Torsten Wiesel and Susumu Tonegawa, laid some ground rules to help OIST succeed.

At least $50 \%$ of researchers at every level - faculty members, postdocs, doctoral students - must be from outside Japan, and English is the official language for everything.

So far, the plan has paid off. OIST began admitting graduate students in 2012, and now has about 60 faculty members - recruited from every continent but Antarctica. It is currently ranked 119 th out of 1,286 academic institutions in the Asia Pacific region and it is 8th among Japanese academic institutions according to its research output, as calculated by the Nature Index.

The founders encouraged diversity of all types - ethnic, gender, disciplinary and academic background. "There was a commitment to diversity with the expectation that it was, not just a nice thing to do, but necessary to the success of the whole thing," says Robert Baughman, executive vicepresident of OIST.

The data support that assertion. Several studies have found that a 
7. greater mix of nationalities and ethnicities correlates with a lift in research 崖. output and with bigger impact through citations. In a 2013 analysis ${ }^{1}$, ü papers generated from the United States and the United Kingdom that is had at least one international author garnered an "impact premium" - a relative bump in the average citation rate - over papers in which all authors were domestic.

A 2014 analysis $^{2}$ looked at 2.5 million US-based papers using surnames as a proxy (albeit a crude one) for ethnicity. Papers with 4 or 5 authors of different ethnicities had 5-10\% more citations on average than papers from authors of all the same ethnicity.

Sowwan's group, which includes ten scientists from Africa, Asia, Australia, Europe and the United States, designs nanoparticles for environmental monitoring and biomedicine, blending materials science, physics, chemistry, nanodevice fabrication and computer simulation.

Sowwan says that the varied backgrounds of the lab members strengthen the group's output, and that he tends to see two tracks of scientists turn up in his lab. Many of the scientists recruited from worldclass universities are 'big picture' thinkers, who, when given a particular research question, return with more excellent questions. The researchers who hail from countries with weaker scientific infrastructures, he says, generally excel at addressing every last detail of the original question.

"They complement each other, and, in the end, they will learn from each other how to always be looking in new directions and at the details," says Sowwan.

In the past 2 years, Sowwan's group has published 14 papers and filed 4 US patents. And six former postdocs have since landed assistant-professor positions at universities around the world.

But the reasons for the connections between diversity and scientific reach and impact are not entirely clear. "It is easy to find correlations, but it is hard to prove causality," says Wei Lee Woon, a computer scientist at Khalifa University of Science \& Technology in Abu Dhabi.

It could be that it is not the diversity breeding the success, but rather that successful labs in top-tier institutions attract a diverse array of good candidates. To unpick the association, Woon and his Khalifa colleagues Talal Rahwan and Bedoor AlShebli probed the Microsoft Academic Graph, a database of scholarly publications. The trio analysed papers published between 1958 and 2009 by universities in the United States, United Kingdom, Canada and Australia ${ }^{4}$, assigning impact to about 1 million papers and their groups of co-authors on the basis of 5 -year citation counts. The data scientists also used authors' surnames to gauge ethnicity, first names to infer gender and first publication date to assess academic age. They also accounted for authors' discipline and affiliation.

When they plotted those scores against the papers' citation counts, the ethnic diversity of the group correlated with impact more strongly than did any other category (see 'Diversity's impact'). But did greater ethnic diversity actually drive citations up? To assess that, the researchers needed a "controlled experiment where we could fix everything else a priori and then 'apply' diversity to a set of papers", says Rahwan.

The team split the papers into those with higher-than-average ethnicdiversity scores and those with lower-than-average scores. Crucially, the researchers ensured that the two sets of papers were similar in terms of publication year, number of authors per paper and the other four types of diversity. They matched 45,689 papers and say that they found that, all other factors being equal, the papers written by ethnically diverse groups were cited $11.2 \%$ more than were papers written by non-diverse groups.

The team also tracked 'individual' diversity, using the ethnic diversity of each author's entire set of collaborators across all papers. Higher individual scores also gave higher citation counts, but group diversity was the bigger driving force behind a paper's impact ${ }^{4}$.

"Ethnic diversity is more about the now - who you are collaborating with right now," on a paper, says AlShebli. Research labs or companies would be wise to hire researchers who complement the group's existing ethnic mix, adds Woon.

The mechanism behind the bump is still elusive. "We've had so many discussions about this," says Woon. It could still be down to high-quality leaders attracting the best people from around the world. Or papers with more international authors could be shared with a wider, more diverse
DIVERSITY'S IMPACT

Ethnic diversity correlates more strongly ( $r$ ) with citation counts than do diversity in age, gender or affiliation, according to an analysis of more than 1 million papers in 24 academic subfields (circles).
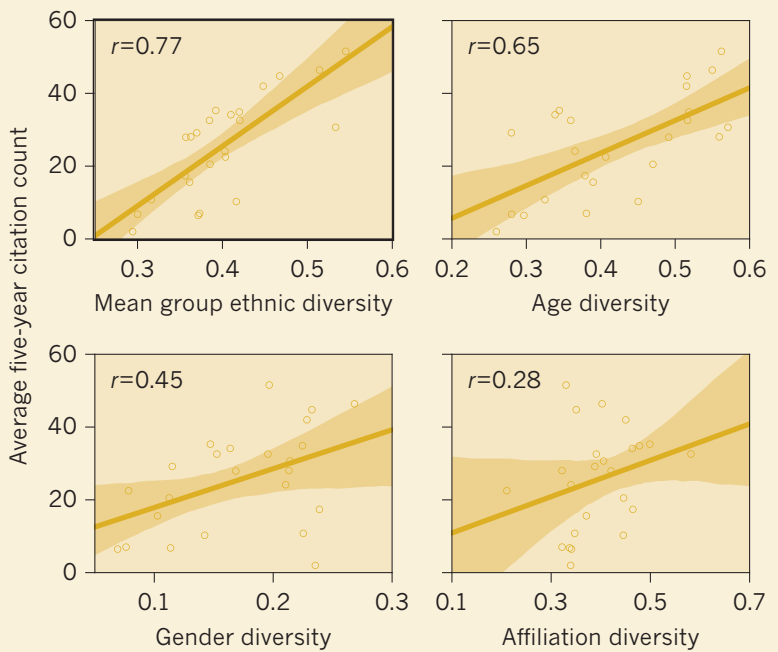

GROUP VERSUS INDIVIDUAL DIVERSITY

Diversity within the list of authors on a paper (group diversity) has a stronger effect on citation count than does individual diversity, the measure of diversity in a researcher's network of collaborators.

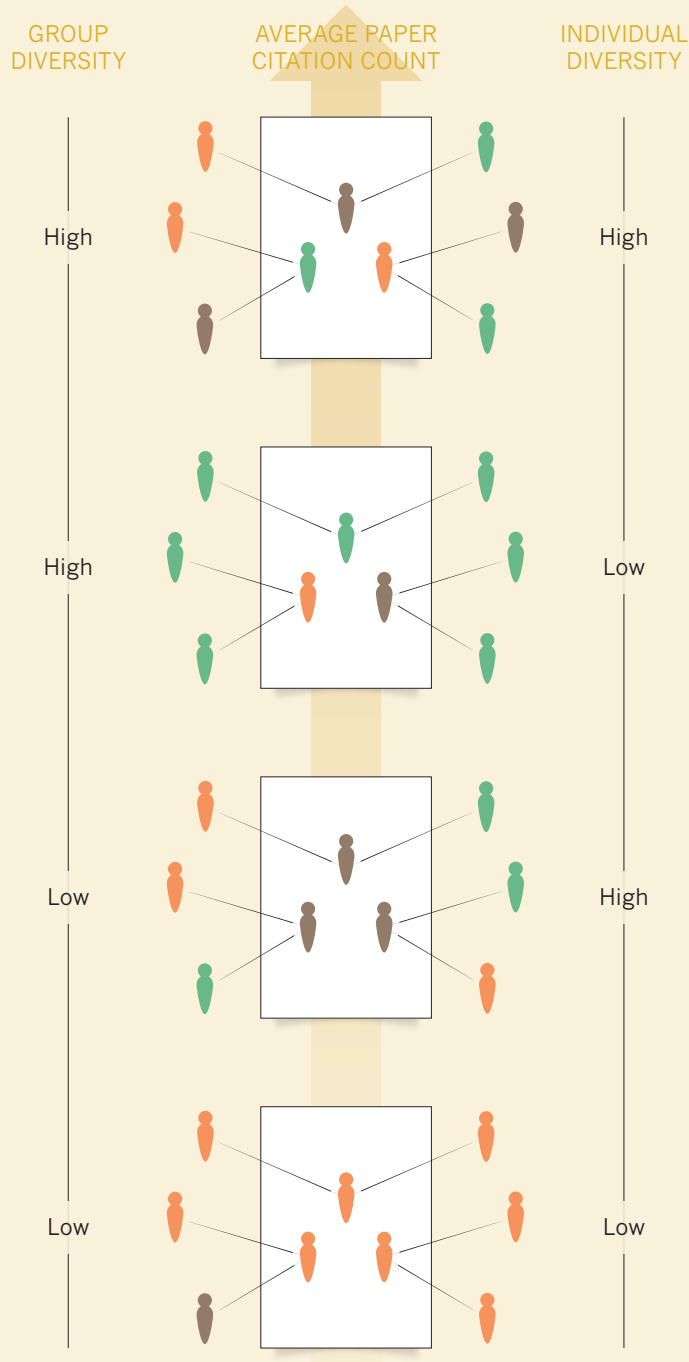




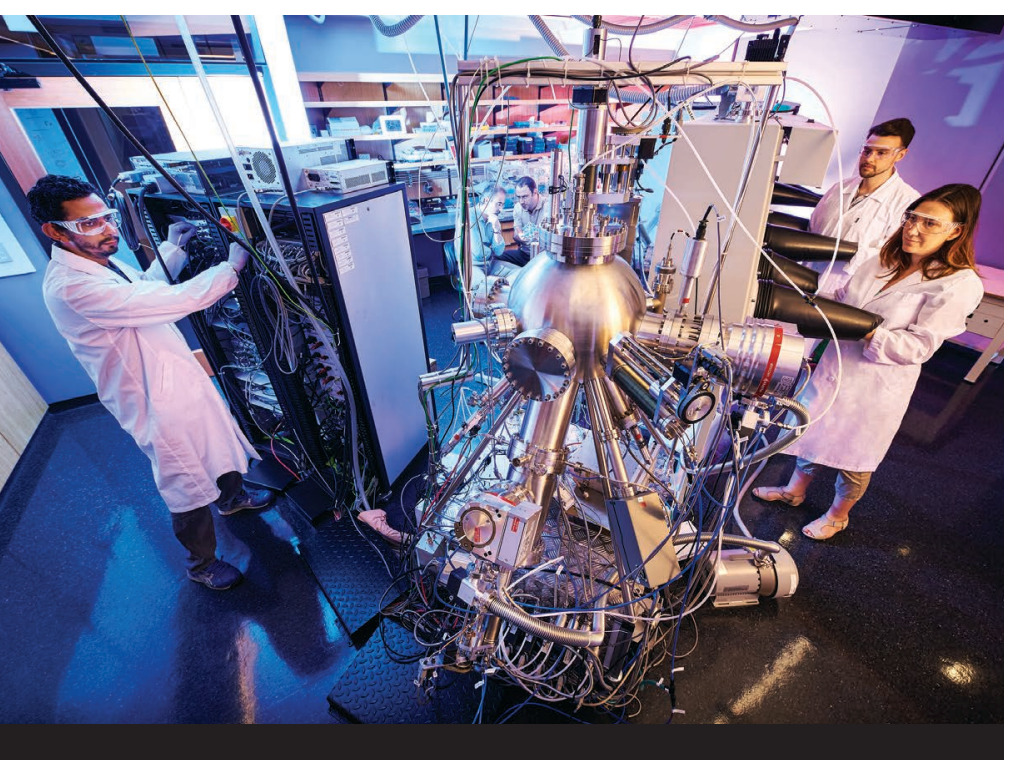

- network, increasing citations.

Woon's team will probe the mechanism of wider networks next. But, Woon says, he would be a little disappointed if that turned out to be the answer to diversity's positive influence. "We all hope it's due to the different cultures cross-fertilizing ideas."

\section{EMBRACING THE SPECTRUM}

In his doctorate work at Vanderbilt University in Nashville, Tennessee, Dave Caudel says, he drew on his unique way of processing the world. As someone with autism spectrum disorder (ASD), he found a welcoming home in the lab of astrophysicist Keivan Stassun, who has been an unflagging advocate of diversity in science. Stassun thinks that diverse perspectives increase the frequency of 'aha' moments.

Caudel finds no reason to argue with that. "It's hard to point to something that was valuable because he was black or she was a Hispanic woman," he says. "But it's no accident that Keivan has published in Nature five times. That's him and his group harnessing all that capability."

Although Caudel had published as an undergraduate researcher, he bombed in the standardized tests needed for entry into graduate school scoring in the bottom $4 \%$. He found his way to Stassun's group through the Fisk-Vanderbilt Bridge Program, an initiative to help under-represented groups succeed in science. The bridge programme "opened a door where there was none", Caudel says.

Stassun's open-door approach springs from his personal background, growing up Mexican American with a single mother and starting his education, he says, from the "bottom of the socio-economic ladder".

Now senior associate dean for graduate education in the College of Arts and Sciences at Vanderbilt, he welcomes young scientists from groups that are under-represented in astrophysics, including women and ethnic minorities, people from low-income families and first-generation university students. Currently, he's mentoring 19 graduate students and postdocs from very different backgrounds.

"I'm intentionally bringing in people from diverse backgrounds and with diverse ways of thinking because it forces us all out of easy assumptions," he says.

Caudel is part of Stassun's efforts to embrace neurodiversity — which describes people whose brains process information differently owing to conditions such as ASD, attention deficit hyperactivity disorder or dyslexia. Once again, the drive to be inclusive stems from Stassun's own experience - he has a son with ASD who dreams of becoming an engineer. "I hope he will enter a world where neurodiversity is something people understand and celebrate."

Caudel relishes working in a lab where he is celebrated for his differences and "given the freedom to work in the way that works best for my brain and how I'm wired". And when Caudel misses a social cue or
Muhkles Sowwan's team includes scientists from five continents. Stassun. Caudel says that he can spend hours, days, weeks or even months thinking obsessively about a problem, "chipping away at the series of thoughts". But he also has times when he gets overloaded with too much sensory input and has to take a day off.

Ironically, Stassun points out, a welcoming, vibrant lab that is socially buzzing and chatty can be difficult for some people with ASD to navigate. So he has come up with different ways for people to participate - such as replacing face-to-face attendance at big group meetings with instant messaging.

The group remains highly productive. It published 47 papers in 2017. Stassun calls it a "well-oiled machine with all the oars pulling in the same direction at the same time".

He points to a 2013 discovery showing that the size and evolutionary stage of a star could be determined by a clever measurement of its flickering starlight ${ }^{5}$. "A multiracial, multigendered group of researchers put our heads together and made this very surprising discovery," he says. "It required a group of very different kinds of people looking at the same data from different perspectives."

\section{CHALLENGES}

Tensions are inevitable when running diverse research teams, where lab mates bump up against each other's cultural differences, language barriers and varying beliefs on what constitutes 'personal space'. In Stassun's group, the level of diversity forces everyone to "take an extra beat" and think twice before speaking in vernacular, short-hand or culturally coded language. Stassun says his biggest hurdle is teaching his lab members to be better, more precise communicators.

"This isn't just me being nice" to guard against misstatements or offensive remarks, he says. "This lab requires precise communication to be at its best."

Some lab heads 'force' their groups to interact socially and scientifically — sometimes by literally rubbing elbows, sitting in close proximity. The Stassun lab has weekly, somewhat mandatory, social gatherings that include food and drink. And Sowwan's team never misses a chance to celebrate a birthday or to share cultural celebrations such as Diwali.

Lab members say that the together time helps to dissolve cultural biases and misunderstandings. The top-down leadership of their lab heads sets the expectation that even if people are uncomfortable at times with diversity, it's worth the fresh perspectives that it brings.

Sowwan sees the frequent, mini culture clashes that happen at OIST as an opportunity to attack the problem of biases from the bottom up. OIST researchers share a camaraderie because "we are all from outside”, says Marta Haro, an electrochemist in Sowwan's lab. Her colleague Zakaria Ziadi, an electrical engineer, says that the atmosphere breeds tolerance. "Working here, you see people becoming more flexible, adaptable and more accepting."

The cohesion of each of these groups embodies something referred to as critical mass - a phenomenon in which under-represented groups experience less stereotyping and higher inclusivity when their numbers reach a certain percentage, typically $15-30 \%$, of the total work group ${ }^{6}$.

More investigators should follow the lead of inclusive labs because the pay-off in scientific impact is real and substantial, says Rahwan. "People need to go beyond their comfort zones, because there is a measurable benefit." - SEE EDITORIAL P.5 AND CAREERS P.149

Kendall Powell is a freelance writer based in Lafayette, Colorado.

1. Adams, J. Nature 497, 557-560 (2013).

2. Freeman, R. B. \& Huang, W. Nature 513, 305 (2014).

3. Burchard, E. G., Oh, S. S., Foreman, M. G. \& Celedón, J. C. Am. J. Respir. Crit. Care Med. 191, 514-521 (2015).

4. AlShebli, B. K., Rahwan, T. \& Woon, W. L. Preprint at https://arxiv.org/ abs/1803.02282 (2018).

5. Bastien, F. A., Stassun, K. G., Basri, G. \& Pepper, J. Nature 500, 427-430 (2013).

6. Nielsen, M. W. et al. Proc. Natl Acad. Sci. USA 114, 1740-1742 (2017). 